Check for updates

Cite this: J. Mater. Chem. C, 2019, 7, 143

Received 1st August 2018,

Accepted 22nd November 2018

DOI: $10.1039 / c 8 t c 03825 b$

rsc.li/materials-c

\section{$13 \mathrm{~mW}$ operation of a 295-310 nm AlGaN UV-B LED with a p-AIGaN transparent contact layer for real world applications $\uparrow$}

\author{
M. Ajmal Khan, (D)*a Noritoshi Maeda, ${ }^{a}$ Masafumi Jo, ${ }^{a}$ Yuki Akamatsu, ${ }^{b}$ \\ Ryohei Tanabe, ${ }^{\mathrm{b}}$ Yoichi Yamadab and Hideki Hirayama ${ }^{\mathrm{a}}$
}

\begin{abstract}
Smart, high-power ultraviolet (UV)-B light-emitting diode (LED) light sources are demanded for both medical and agricultural applications, including vitamin D3 production in human skin (294-304 nm), immunotherapy $(310 \mathrm{~nm})$, cancer therapy $(295-310 \mathrm{~nm})$ and enriching phytochemicals in plants $(310 \mathrm{~nm})$. To achieve this, we have combined graded stacks of AlGaN buffer layer (BL), AlGaN multi quantum wells (MQWs) with high internal quantum efficiency (IQE), a transparent p-AIGaN contact layer, and a highlyreflective $p$-type electrode for the fabrication of a UV-B LED. By optimizing the growth conditions, we demonstrated an output power of $7.1 \mathrm{~mW}$ for a $310 \mathrm{~nm}$ UV-B LED under bare-wafer conditions using a highly reflective $\mathrm{Ni} / \mathrm{Mg} \mathrm{p}$-electrode. We also demonstrated a high IQE of 47\% for UV-B emission from UV-B LED at $295 \mathrm{~nm}$, by using a graded n-AIGaN BL. The light-extraction efficiency (LEE) was increased by introducing both a highly-transparent $\mathrm{p}-\mathrm{AlGaN}$ and a highly reflective $\mathrm{Ni} / \mathrm{Mg} \mathrm{p}$-electrode. As a result, we achieved an EQE of $4.4 \%$ at a dc drive current of $20 \mathrm{~mA}$ under CW-operation at RT and a maximum output power of $13 \mathrm{~mW}$ for a $295 \mathrm{~nm}$ UV-B LED for medical applications.
\end{abstract}

\section{Introduction}

The recent demand for smart, cheap and environmentally safe AlGaN ultraviolet light-emitting diodes (UV-LEDs) with wavelengths below $350 \mathrm{~nm}$ has arisen due to numerous potential applications, such as those in immunotherapy $(310 \mathrm{~nm})$, for vulgaris treatment $(310 \mathrm{~nm})$, gravitational sensors, plant lighting $(310 \mathrm{~nm})$, water sterilization, assisting in the transportation of foods, the manufacture of bank notes, the production of vitamin D3 in the human body (294-304 nm), the detection of biochemical agents, fluorescence detection, and the identification of proteins, DNA, and bacteria. ${ }^{1-6}$

Our principal focus in this work was on the crystal growth of AlGaN UV-B LEDs for both $295 \mathrm{~nm}$ and $310 \mathrm{~nm}$ emission and the evaluation of their performances for certain medical and agricultural applications. A brief review of the use of UV light in agricultural and medical applications is called for. UV-B light can penetrate only the epidermis of human skin and the most superficial parts of the rete ridges, whereas UV-A light sources

\footnotetext{
${ }^{a}$ Quantum Optodevice Laboratory, RIKEN, 2-1 Hirosawa, Wako, Saitama, 351-0198, Japan.E-mail: muhammad.khan@riken.jp

${ }^{b}$ Faculty of Engineering, Yamaguchi University, 2-16-1 Tokiwadai, Ube, Yamaguchi, 755-8611, Japan

$\dagger$ Electronic supplementary information (ESI) available. See DOI: 10.1039/ c8tc03825b
}

can penetrate deeper into human skin and possibly reach the vascular bed, and, therefore, UV-A is not suitable for the treatment of psoriasis. ${ }^{5,7}$ Broadband BB-UVB (280-320 nm) therapy was found to be less effective than narrowband UV-B at $310 \mathrm{~nm}$ emission in the treatment of psoriasis and atopic dermatitis because the risk of erythema and sunburn of the non-affected skin was higher. ${ }^{8,9}$ Therefore, narrowband $(310 \mathrm{~nm})$ UV-B phototherapy can be used to cure cancer and skin diseases while minimizing the adverse side effects on neighboring non-affected tissues. ${ }^{10-15}$ Narrowband UV-B light sources were highly recommended for the treatment of psoriasis in the national guidelines of the USA and also in some other countries too. ${ }^{8,16,17}$ Torii et al. used stratified normal human keratinocytes $(\mathrm{sNHK})^{13}$ and then the effects of UV-B light with an emission wavelength of $280 \mathrm{~nm}$ and $310 \mathrm{~nm}$ on the apoptosis (death of the cells) of HaCaT cells were compared, as shown in Fig. 2(a) of ref. 13. It was confirmed that $310 \mathrm{~nm}$ UV-B induces the secretion of high-mobility group box-1 (HMGB1) ${ }^{12,13}$ without inducing apoptosis, but on the other hand, $280 \mathrm{~nm}$-band UVB induces secretion of HMGB1 and apoptosis (death of the cells). Unfortunately, semiconductor devices designed for phototherapy using UV-B LEDs are still very rare $^{5}$ and therefore few clinical studies have been done so far using LED light sources for dermatological applications. ${ }^{14,15}$

In the case of agricultural applications, recently, it was discovered that the phytochemicals in fruit and vegetables can be 
enriched by using narrowband-310 nm UV-B light sources, even with a modest output power of around $20 \mathrm{~mW}$. Phytochemicals from plants can greatly reduce the risk of cancer and cardiovascular disease, ${ }^{5}$ demonstrating an indirect medical application of narrowband UV-B LED to further improve quality of life (QOL). One important result in the application of UV-B LEDs came from Boston University in the USA, where it was discovered that a UV-B LED with an emission wavelength of $293 \mathrm{~nm}$ is 2.4 times more efficient than sunlight for producing vitamin D3 in the human body. ${ }^{18}$

Based on this comparative review study of UV-B light sources, we picked out two important UV-B-wavelength emission applications between $294 \mathrm{~nm}$ and $310 \mathrm{~nm}$. Therefore, we began our research and development of UV-B LED devices here at Riken, with medical science (294-310 nm) and agricultural applications (310 nm) in mind, in 2017. Recently, our group reported an AlGaN UVC LED that uses a highly reflective photonic crystal (PhC) on a p-AlGaN contact layer, with which an EQE of $10 \%$ was successfully achieved. ${ }^{19}$ Our group, in collaboration with Panasonic Corporation, successfully achieved a world record EQE of $20 \%$ from an experimental AlGaN based UVC-LED grown on an AlN buffer layer on a patterned sapphire substrate (PSS). ${ }^{20}$ UV Craftory, Nitek, and Nichia have also developed some DUV LEDs using AlGaN or InAlGaN. ${ }^{5,6,21-24}$ However, the performance of the UV-B LED devices based on AlGaN on AlN/sapphire templates is still very low. ${ }^{5,6}$ Kim et al. fabricated AlGaN based UV-B LEDs grown on an AlN epilayer on a sapphire substrate and reported emission at $290 \mathrm{~nm}$ with a maximum output power of around $1.8 \mathrm{~mW}$, and emission at $305 \mathrm{~nm}$ with a maximum output power of around $2.7 \mathrm{~mW} .{ }^{25} \mathrm{In}$ 2017, Susilo et al. of TU Berlin demonstrated an output power of $0.83 \mathrm{~mW}$ at $302 \mathrm{~nm}$ with a wall plug efficiency of $0.4 \%$ at $20 \mathrm{~mA}$, from AlGaN UV-B LEDs grown on an AlN template. ${ }^{26}$ A p-GaN contact layer is unsuitable for UV-B emission wavelengths and p-AlGaN is suitable for UV-B LEDs. ${ }^{6,27}$ Therefore, very few reports are available on the subject of AlGaN based UV-B LED devices on AlN/sapphire templates with 294-310 nm emission using highly transparent p-AlGaN layers instead of p-GaN as well as using a highly reflective p-electrode. However, there is scope for improving the performance of AlGaN devices and to fabricate smart, cheap and environmentally safe UV-B LEDs for both agricultural and medical applications with emission wavelengths of $294 \mathrm{~nm}$ and $310 \mathrm{~nm}$ respectively. Recent developments in AlN/AlGaN/MQW UV LED crystal growth technology have led to an IQE of more than $60 \%$ for those grown on AlN templates ${ }^{5}$ and more than $90 \%$ for those grown on a single crystal AlN substrate. ${ }^{6}$ In the case of UVC LEDs, the electron injection efficiency (EIE) was increased to $80 \%$ by employing a multi-quantum blocking (MQB). ${ }^{5,6}$ However, the LEE of our UV LED devices is still under $10 \% .^{5,6,19,20,28,29}$ The EIE of the $310 \mathrm{~nm}$-band UV-B LED is also very low. It is therefore necessary to further improve LEE and EIE in the AlGaN UV-B LED devices. Therefore, we attempted to fabricate devices both for $295 \mathrm{~nm}$ emission, which can be used to produce vitamin D3 in the human body, and also for $310 \mathrm{~nm}$-emission, which is the ideal wavelength to induce HBGT-1 in the human body to treat psoriasis and cancer, as well as for enriching phytochemicals in plants. In this paper, we aimed to achieve a novel crystal growth technique with which we can produce high-quality graded stacks of AlGaN BL (with low TDDs), deposit AlGaN based MQWs with a high IQE at $310 \mathrm{~nm}$ emission, and also fabricate highly transparent p-AlGaN contact layers, as compared to the conventional UV-B LEDs. We also aimed to achieve highly reflective p-electrodes deposited on the front side of both UV-B LED devices with emission wavelengths of $310 \mathrm{~nm}$ and $293 \mathrm{~nm}$, respectively.

\section{Experiment}

In order to obtain AlGaN based UV-B LED devices with IQEs above 70 or $80 \%$, an AlN template/sapphire with a TDD of $10^{8}-10^{7} \mathrm{~cm}^{-2}$ is needed..$^{5,6,30,31}$ To achieve this goal, our group implemented an "ammonia $\left(\mathrm{NH}_{3}\right)$ pulsed-flow multilayer $(\mathrm{ML})$ growth technique", with which good quality AlN layers grown on a (0001)-sapphire substrate, having FWHM values of the XRCs for the (0002) and (10-12) planes of 200 and 300 arcsec, respectively (TDD $\sim 1 \times 10^{8} \mathrm{~cm}^{-2}$ ), could be obtained. . $^{5,19,20,31}$ In this work, low-pressure metalorganic vapor-phase epitaxy (LP-MOVPE), with trimethylgallium (TMGa) and trimethylaluminum (TMAl) as group-III precursors, and ammonia $\left(\mathrm{NH}_{3}\right)$ for the group-V element, was used to grow UV-B LED heterostructures on AlN templates grown on (0001)-oriented $c$-plane sapphire substrates. Tetraethylsilane (TESi) and bis(ethylcyclopentadienyl)magnesium $\left(\mathrm{Cp}_{2} \mathrm{Mg}\right)$ were used as n- and p-type doping sources, respectively. Previously, a 2-stack n-AlGaN BL based conventional UV-B LED structure with an emission wavelength of 310-315 $\mathrm{nm}$ was attempted. First, stacking layers of an approximately $1 \mu$ m-thick Si-doped $n-\mathrm{Al}_{0.60} \mathrm{Ga}_{0.40} \mathrm{~N}$ BL and then $1 \mu$ m-thick Si-doped $\mathrm{n}-\mathrm{Al}_{0.36} \mathrm{Ga}_{0.64} \mathrm{~N}$ CSL were grown epitaxially on the overlayer of an approximately $4 \mu \mathrm{m}$-thick AlN template on a $400 \mu \mathrm{m}$-thick $c$-plane-(0001)-sapphire substrate at the growth temperature of $1130{ }^{\circ} \mathrm{C}$ under a pressure of 76 Torr. Next, an overlayer of the active region (MQWs) consisting of a 3-fold $\mathrm{Al}_{0.25} \mathrm{Ga}_{0.75} \mathrm{~N}(4-5 \mathrm{~nm}) / \mathrm{Al}_{0.36} \mathrm{Ga}_{0.64} \mathrm{~N}(15-20 \mathrm{~nm})$ structure was deposited on the n-AlGaN CSL. Subsequently, 2-fold lightly Mg-doped $\mathrm{p}-\mathrm{Al}_{0.40} \mathrm{Ga}_{0.60} \mathrm{~N}$ layers $(60 \mathrm{~nm})$ were inserted as MQB layers between the MQWs and p-AlGaN cladding layers. Finally, an approximately $150 \mathrm{~nm}$ moderately $\mathrm{Mg}$-doped p- $\mathrm{Al}_{0.40} \mathrm{Ga}_{0.60} \mathrm{~N}$ cladding layer and an approximately $20 \mathrm{~nm}$ heavily $\mathrm{Mg}$-doped $\mathrm{p}^{+}-\mathrm{Al}_{0.40} \mathrm{Ga}_{0.60} \mathrm{~N}$ contact layer, respectively, were grown. A schematic structure of the AlGaN based conventional UVB LED (sample-R) is shown in Fig. 1(a). Secondly, a $310 \mathrm{~nm}$ UV-B LED sample-A at growth temperature $1135-1140{ }^{\circ} \mathrm{C}$ was also attempted. The $310 \mathrm{~nm}$-band UV-B LED (sample-A) consisted of an approximately $4 \mu \mathrm{m}$-thick undoped AlN template on a sapphire substrate, an approximate total of $2.5 \mu \mathrm{m}$-thick fourstack of Si-doped n-Al $\mathrm{Al}_{0.60} \mathrm{Ga}_{0.40} \mathrm{~N}$ BL-1 $(1.9 \mu \mathrm{m}), \mathrm{n}-\mathrm{Al}_{0.52} \mathrm{Ga}_{0.48} \mathrm{~N}$ BL-2 (300 nm), n-Al ${ }_{0.47} \mathrm{Ga}_{0.53} \mathrm{~N}$ BL-3 (150 nm) and n- $\mathrm{Al}_{0.42} \mathrm{Ga}_{0.58} \mathrm{~N}$ CSL $(150 \mathrm{~nm})$, a three-fold MQW emitting region consisting of approximately $5 \mathrm{~nm}$-thick $\mathrm{Al}_{0.32} \mathrm{Ga}_{0.68} \mathrm{~N}$ wells and $20 \mathrm{~nm}$-thick $\mathrm{Al}_{0.42} \mathrm{Ga}_{0.58} \mathrm{~N}$ barrier layers $(310 \mathrm{~nm})$, a $62 \mathrm{~nm}$-thick 2-fold 
(a)

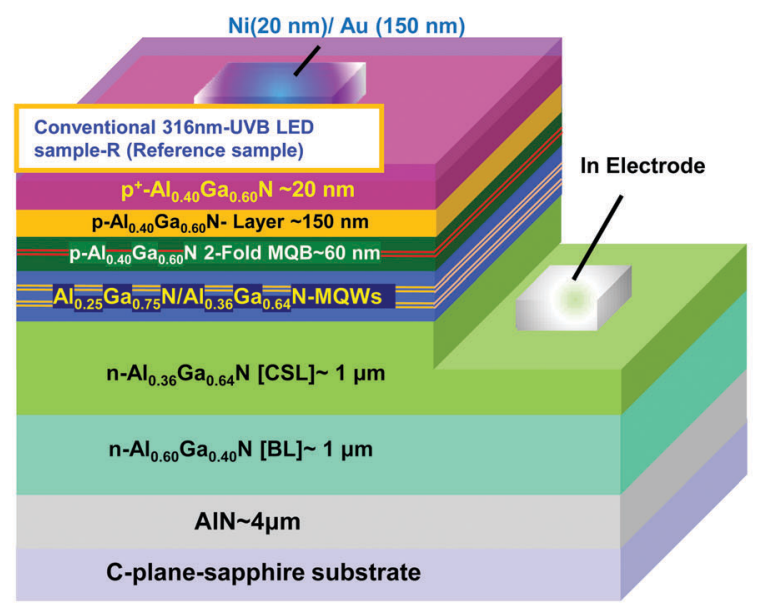

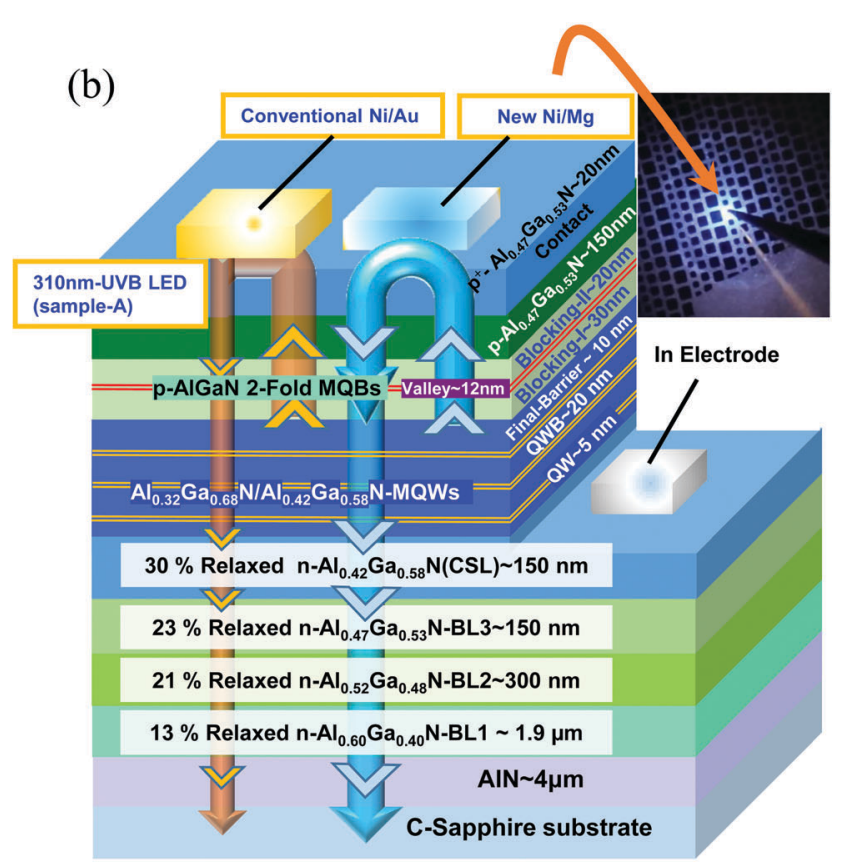

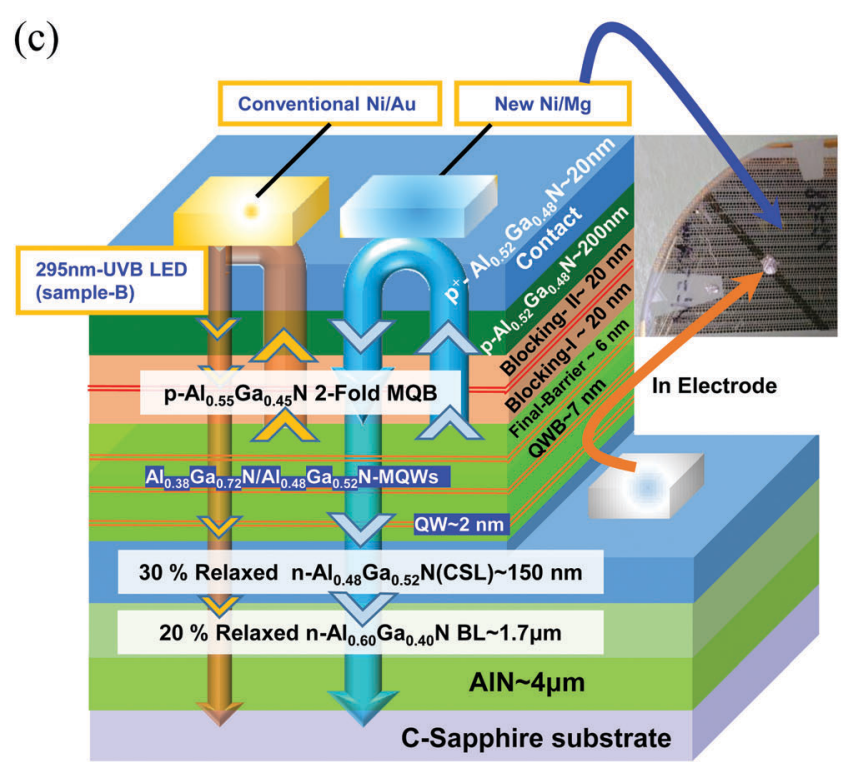

Fig. 1 (a) Schematic of 2-stack conventional AIGaN BL based $316 \mathrm{~nm}$ UV-B LEDs, with a conventional Ni/Au p-electrode (sample-R), (b) schematic of 4-stack AIGaN BL based $310 \mathrm{~nm}$ UV-B LEDs, one with a conventional Ni/Au p-electrode and the other one with a highly-reflective Ni/Mg p-electrode (the real image of $310 \mathrm{~nm}$ UVB LED device under operation with p-electrode is shown in the inset), and (c) schematic of 2-stack AIGaN BL based $295 \mathrm{~nm}$ UV-B LEDs, one with a conventional Ni/Au p-electrode and the other one with a highly-reflective Ni/Mg $\mathrm{p}$-electrode (the real image of $295 \mathrm{~nm}$ UVB LED with both In-dot n-electrode and $p$-electrode is shown in the inset).

Mg-doped AlGaN/AlGaN MQB, and an approximately $150 \mathrm{~nm}$ thick Mg-doped $\mathrm{p}-\mathrm{Al}_{0.47} \mathrm{Ga}_{0.53} \mathrm{~N}$ layer including a $\mathrm{p}$-AlGaN p-contact layer $(20 \mathrm{~nm})$. The typical structure of the UV-B LED (sample-A), with emission wavelength of $310 \mathrm{~nm}$, is shown in Fig. 1(b).

Finally, the 295 nm-band UV-B LED (sample-B) consisted of an approximately $4 \mu \mathrm{m}$-thick undoped AlN template on a sapphire substrate, a total $1.8 \mu \mathrm{m}$-thick two-stack of Si-doped $\mathrm{Al}_{0.60} \mathrm{Ga}_{0.40} \mathrm{~N}$ BL $(1.7 \mu \mathrm{m})$, including $\mathrm{n}-\mathrm{Al}_{0.48} \mathrm{Ga}_{0.52} \mathrm{~N}$ CSL (150 nm), a 3-fold MQW emitting region consisting of approximately $2 \mathrm{~nm}$-thick
$\mathrm{Al}_{0.38} \mathrm{Ga}_{0.72} \mathrm{~N}$ wells and $7 \mathrm{~nm}$-thick $\mathrm{Al}_{0.48} \mathrm{Ga}_{0.52} \mathrm{~N}$ quantum barrier layers, a $60 \mathrm{~nm}$-thick 2-fold Mg-doped AlGaN/AlGaN MQB, and an approximately $150 \mathrm{~nm}$-thick Mg-doped $\mathrm{p}-\mathrm{Al}_{0.52} \mathrm{Ga}_{0.48} \mathrm{~N}$ layer including a p-AlGaN contact layer $(20 \mathrm{~nm})$. The typical structure of the UV-B LED (sample-B), with an emission wavelength of $295 \mathrm{~nm}$, is shown in Fig. 1(c).

The structural properties, crystallinity, compositions, strainrelaxation rate and threading dislocation densities (TDDs) in the n-AlGaN layer and the LED heterostructures were investigated by X-ray rocking curve (XRC) as well as reciprocal space 
mapping (RSM) analysis and TEM observation, including crosssectional high-angle annular dark field scanning transmission electron microscopy (HAADF-STEM). In the present case, for sample-B of $295 \mathrm{~nm}$ emission wavelength, we used the second harmonic light from a dye laser pumped by a Xe-Cl excimer laser as an excitation source for standard IQE measurement by temperature-dependent and excitation-power-density-dependent PL spectroscopy. ${ }^{33}$ The pulse width and repetition rate were $20 \mathrm{~ns}$ and $100 \mathrm{~Hz}$, respectively. The excitation photon energy was $5.164 \mathrm{eV}(240 \mathrm{~nm})$. Two different types of p-electrode were used. On some devices, conventional $20 \mathrm{~nm}$ thick Ni followed by $150 \mathrm{~nm}$ thick $\mathrm{Au}$, which has a low reflectance, was used, while on others, highly reflective $1 \mathrm{~nm}$ thick Ni followed by $200 \mathrm{~nm}$ thick $\mathrm{Mg}$ was used, as shown in Fig. 1(a)-(c), respectively. Subsequently, rapid thermal annealing at $550{ }^{\circ} \mathrm{C}$ for $5 \mathrm{~min}$ was done. The Indium dot (In-dot) metal deposition technique was used for the fabrication of $n$-electrodes after exposing a $2 \mathrm{~mm} \times 2 \mathrm{~mm}$ area of the wafer by a mechanical method and subsequently the In-dot was deposited on the exposed area for quick check measurement, as shown in the inset of Fig. 1(c). Measurements of the device characteristics were done at room temperature (RT) under continuous wave (CW)-operation. The size of the p-type electrodes was $0.25 \times 0.25 \mathrm{~mm}^{2}$, as shown in the inset of Fig. 1(b). The output power was measured under bare-wafer conditions using a Si photodetector located behind the sample. This was calibrated to measure the luminous flux in order to give accurate values for the output power. Current $v s$. output power $(I-L)$, current $v s$. voltage $(I-V)$ and current $v s$. EQE $(I-\mathrm{EQE})$ measurements were performed under $\mathrm{CW}$-operation at RT.

\section{Results and discussion}

Schematic illustrations showing the structure of the grown devices with a conventional Ni/Au p-electrode and with a new (highly-reflective) $\mathrm{Ni} / \mathrm{Mg}$ p-electrode including the conventional UV-B LED structure respectively are given in Fig. 1(a)-(c). The molar ratio of $\mathrm{Al}$ in the $\mathrm{p}$ - and $\mathrm{n}$-cladding/blocking layers should be higher than that in the active layer, but at the same time, a high $\mathrm{Al}$ content becomes a reason for poor $\mathrm{p}$ - and n-type conductivity in the cladding/blocking layers, which in turn degrades the current injection performance across the device structure $^{5,6,32}$ In this work, a high relative transmittance of more than $90 \%$ in the range from $290 \mathrm{~nm}$ to $320 \mathrm{~nm}$ from a $295 \mathrm{~nm}$ UV-B LED (sample-B) was confirmed, as shown in Fig. 2, where the relative transmittance is defined by the ratio of the light intensity transmitted through an LED on an AlN/ sapphire template divided by that through the AlN/sapphire template only. ${ }^{27}$ It is well known that n-AlGaN epitaxially grown on an AlN template/sapphire can have a lattice mismatch as large as $4 \%$, depending on the molar ratio of the aluminum contents. $\mathrm{Al}_{x} \mathrm{Ga}_{1-x} \mathrm{~N}$ with $x \sim 0.65$ may result in $\sim 1 \%$ lattice mismatch at the $\mathrm{Al}_{x} \mathrm{Ga}_{1-x} \mathrm{~N} / \mathrm{AlN}$ interface and a refractive index difference of $\sim 0.1$ between these two layers. But, in the case of $x \sim 0.44-0.56$, the mismatch is $>1.5 \%$ at the $\mathrm{Al}_{x} \mathrm{Ga}_{1-x} \mathrm{~N} / \mathrm{AlN}$ interface. This lattice mismatch causes the storage of elastic

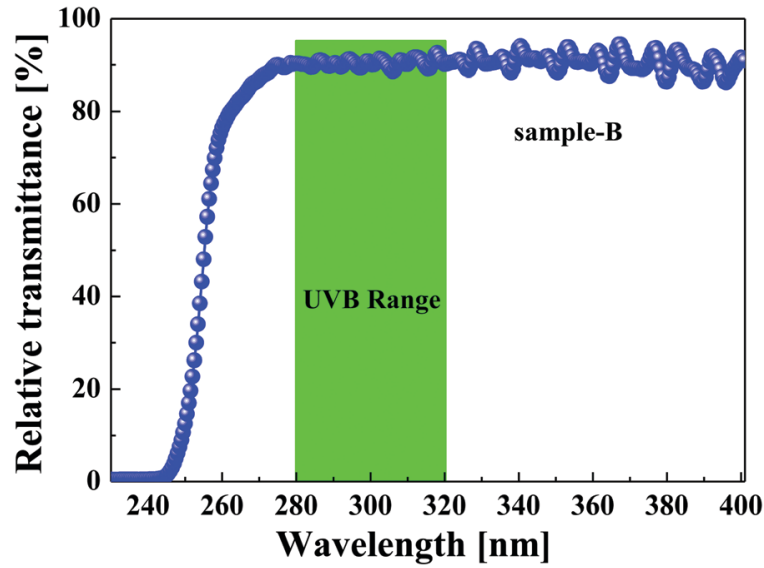

Fig. 2 Relative transmittance through a p-AIGaN UV-B LED (sample-B).

strain energy and this increases as the thicknesses of the epitaxial AlGaN and n-AlGaN layers are increased, as shown in Fig. 3(a) and (b) for sample-A and sample-B, respectively. The magnified RSM image of sample-A is shown in Fig. 3(a), which indicates that the first n-type $\mathrm{Al}_{0.60} \mathrm{Ga}_{0.40} \mathrm{~N}$ BL-1 is in a relaxed state (with a relaxation ratio of $12 \%$ ), with respect to the fully relaxed AlN substrate and similarly the second n-type $\mathrm{Al}_{0.52} \mathrm{Ga}_{0.48} \mathrm{~N}$ BL-2 is in a relaxed state (with a relaxation ratio of $21 \%$ ) with respect to the fully relaxed AlN substrate. The third n-type $\mathrm{Al}_{0.47} \mathrm{Ga}_{0.53} \mathrm{~N}$ BL-3 is in a relaxed state (with a relaxation ratio of $23 \%$ ), with respect to the fully relaxed AlN substrate, and the final n-type $\mathrm{Al}_{0.42} \mathrm{Ga}_{0.58} \mathrm{~N}$ CSL is in a relaxed state (with a relaxation ratio of $30 \%$ ), with respect to the fully relaxed AlN substrate, as shown in Fig. 3(a), and the same is shown in Fig. 1(b). Similarly, the magnified image of RMS in Fig. 3(b) of sample-B indicates that the first n-type $\mathrm{Al}_{0.60} \mathrm{Ga}_{0.40} \mathrm{~N}$ BL is in a relaxed state (with a relaxation ratio of $20 \%$ ), with respect to the fully relaxed AlN substrate, and similarly, the second n-type $\mathrm{Al}_{0.48} \mathrm{Ga}_{0.52} \mathrm{~N}$ CSL is in a relaxed state (with a relaxation ratio of $30 \%$ ) with respect to the fully relaxed AlN substrate. After suitable optimization of the $\mathrm{n}$-AlGaN BL including CSL and MQWs, single peak photoluminescence (PL) emission at the desired targets for UV-B LEDs was successfully achieved, as given in the ESI $\dagger$ (please see Fig. SI(b)). Subsequently, IQEs of around $45-50 \%$ were confirmed for UV-B with single peak PL emission from the MQWs (sample-A and sample-B), respectively. The IQE is also strongly dependent on the crystalline quality of the MQW region, which is further dependent on the crystalline quality of the bottom n-AlGaN CSL.

The film layer thicknesses and dislocation densities of the UV-B LED structure (sample-A) were evaluated using HR-TEM, as shown in Fig. 4(a)-(c). In order to see all types of dislocation densities in the n-AlGaN BL and n-AlGaN CSL, respectively, of sample-A, the electron incidence direction in the image of Fig. 4(a) and in the image of Fig. 4(b) was set to the $\langle 10-10\rangle$ zone axis. Fig. 4(a) and (b) include both $g=$ [0002] (screw type) and $g=[10-20](\mathrm{a}+\mathrm{b}$; edge and mixed types) vector information, respectively, for the estimation of all types of TDDs. The evaluated TDD types and values both for the right side and left 


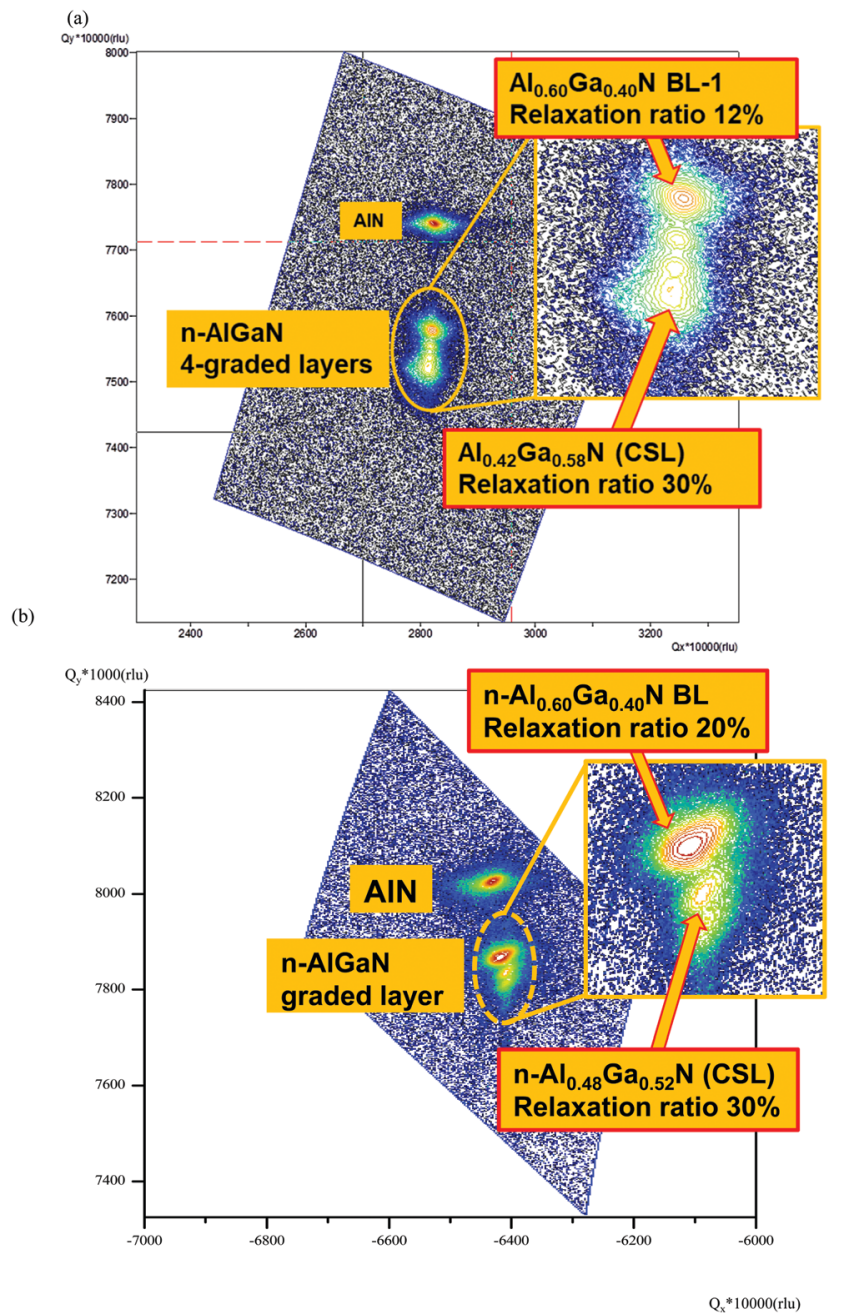

Fig. 3 (a) A reciprocal space map (RSM) for the (105) reflections of $\mathrm{n}$-AIGaN CSL and graded BL, where strain-relaxation state and compositional information is shown in the inset of enlarged image (sample-A), and (b) a reciprocal space map (RSM) for the (105) reflections of $n$-AIGaN CSL and graded $\mathrm{BL}$, where strain-relaxation state and compositional information is shown in the inset of enlarged image (sample-B).

side of the sample-A around the CSL are shown in Fig. 4(a) and given in Table 1 . The total TDDs on the left side field were estimated to be $1.4 \times 10^{9} \mathrm{~cm}^{-2}$ and quite low total TDDs on the right side field in the $\mathrm{n}$-AlGaN CSL were estimated to be $1.1 \times$ $10^{9} \mathrm{~cm}^{-2}$, as given in Table 1 . Such reductions of the TDDs might be caused by a slightly relaxed (20-30\%) n-AlGaN BL and n-AlGaN CSL, as shown by the RSM data of Fig. 3(a). Tilting in the (0002) zone axis visualizes any screw-type dislocations present in the cross-sectioned part of the n-AlGaN BL and n-AlGaN CSL, as can be seen in Fig. 4(a). The screw type TDDs is $5.5 \times 10^{7} \mathrm{~cm}^{-2}$, as given in Table 1 . The result of the n-AlGaN CSL with a low TDD of $1.1 \times 10^{9} \mathrm{~cm}^{-2}$ of sample-A shows a promising route for further improvement of the performance of the $310 \mathrm{~nm}$-band UV-B LED devices. The thicknesses of the graded Si-doped n-AlGaN BL-1, BL-2, BL-3 and CSL were measured to be approximately $2 \mu \mathrm{m}, 300 \mathrm{~nm}, 150 \mathrm{~nm}$ and $150 \mathrm{~nm}$, respectively, as shown in the HAADF-STEM and also in the annular bright-field (ABF)-STEM images of Fig. 4(a)-(c). The thicknesses of the quantum barrier layer, quantum well and final barrier, respectively, were measured to be $20 \mathrm{~nm}, 5 \mathrm{~nm}$ and $10 \mathrm{~nm}$, as shown in the ABF-STEM image of Fig. 4(c), taken from point "P1" of the HAADF-STEM image of Fig. 4(a). The thicknesses of electron blocking-1, valley and electron blocking-2 of the MQB structure, respectively, were also measured to be $30 \mathrm{~nm}, 5 \mathrm{~nm}$ and $20 \mathrm{~nm}$, as shown in the ABF-STEM image of Fig. 4(c).

For comparison, electrical characterization of a conventional UV-B LED (sample-R) having an n-AlGaN/AlGaN/p-AlGaN semiconductor $\mathrm{p}-\mathrm{n}$ junction is discussed briefly, as shown in Fig. 5(a)-(c), along with the new UV-B LED device (sample-A). First, electrical characterization of the conventional UV-B LED (sample-R), with an emission wavelength of $316 \mathrm{~nm}$, using a $\mathrm{Ni} / \mathrm{Au}$ p-electrode was demonstrated, as shown in the dotted line of Fig. 5. During the $I-V$ characterization, the turn on voltage of $6.0 \mathrm{~V}$ at $3 \mathrm{~mA}$ was measured, as shown in the inset of Fig. 5(a). The maximum output power of $3 \mathrm{~mW}$ at $180 \mathrm{~mA}$ using a Ni $(20 \mathrm{~nm}) / \mathrm{Au}(150 \mathrm{~nm})$ p-electrode was demonstrated, as shown in Fig. 5(b). This low output power is attributed to both an un-optimized n-AlGaN BL including MQWs and the possibility of extended defects and crystal irregularities that cannot be ignored in the n-AlGaN CSL of sample-R due to the abrupt change of Ga content from $4 \mathrm{sccm}$ to $9 \mathrm{sccm}$. Therefore, an alternative approach of a 4-stack graded n-AlGaN based BL was proposed in this work to improve the crystallinity of the n-AlGaN CSL beneath the MQWs, as shown in the schematic of Fig. 1(b). A $310 \mathrm{~nm}$-band UV-B LED sample-A with a conventional Ni/Au p-electrode with a single peak emission at $310 \mathrm{~nm}$ has been demonstrated, as shown in Fig. 5(a)-(c). The maximum output power was improved from $3 \mathrm{~mW}$ (sample-R) to $6.5 \mathrm{~mW}$ (sample-A) and similarly the EQE was also improved from $0.4 \%$ to $0.7 \%$, respectively, under bare-wafer conditions under CW-operation at RT, as shown in Fig. 5(b) and (c). Furthermore, the UV-B devices suffer from high resistances during operation due to poor hole injection and transport in the p-type layers, as shown in the $I-V$ characteristics of Fig. 5(a). Typically, a thick p-GaN contact layer is grown on top of the p-AlGaN cladding layer to manage good ohmic contact and hole injection. However, both the p-GaN contact layer and the p-type contact metal (e.g., a Ni- or Au-based metal stack) can cause internal light absorption, and consequently, the light extraction efficiency (LEE) can be degraded. Previously, we increased the EQE of UVC-LEDs by a factor of 1.6 just by replacing the Ni or $\mathrm{Ni} / \mathrm{Au}$ p-electrode with either highly reflective $\mathrm{Rh}$ or $\mathrm{Ni} / \mathrm{Mg}$ p-electrodes. $^{20,28,29}$ Therefore, we attempted to evaporate $\mathrm{Ni} / \mathrm{Au}$ and $\mathrm{Ni} / \mathrm{Mg}$ p-type electrodes onto the p-AlGaN contact layers of the UV-B LEDs, where the thicknesses of the conventional $\mathrm{Ni} / \mathrm{Au}$ electrodes were $20 \mathrm{~nm}$ and $200 \mathrm{~nm}$, and thicknesses of the new $\mathrm{Ni}$ and $\mathrm{Mg}$ stacking layers in the $\mathrm{Ni} / \mathrm{Mg}$ electrodes were approximately $1.0 \mathrm{~nm}$, and $200 \mathrm{~nm}$, respectively, as illustrated in the ESI $\dagger$ (please see Fig. SII(a) and (b)). The emitted light from the QW was absorbed by the $20 \mathrm{~nm}$ thick Ni electrode and $150 \mathrm{~nm}$ thick $\mathrm{Au}$ electrode, due to the low reflectance of the Ni layer (approximately 30\%) and low reflectance of the 
(a) Red: Edge defect Blue: Screw type Purple: Mixed defe

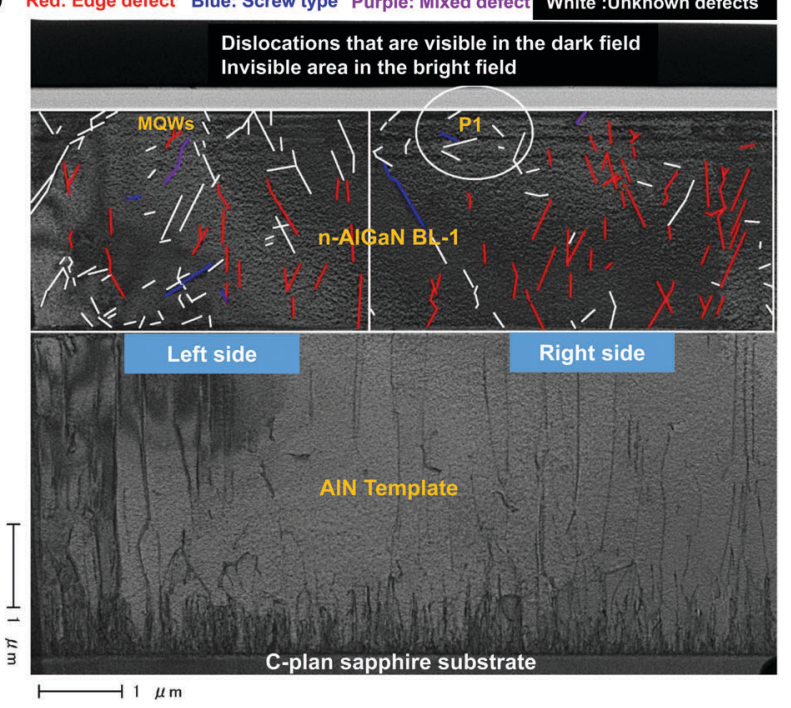

(b)

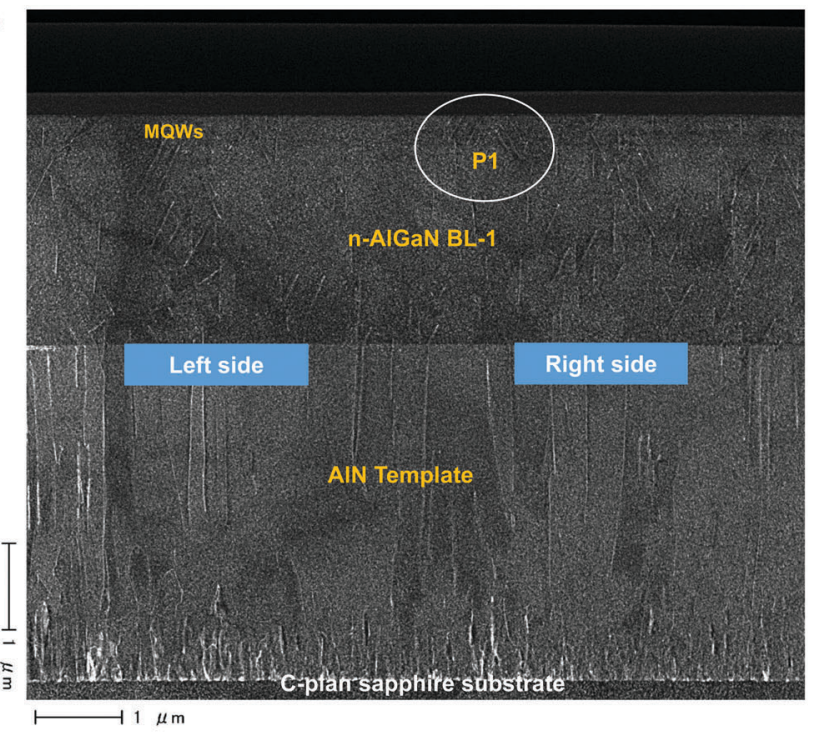

(c)

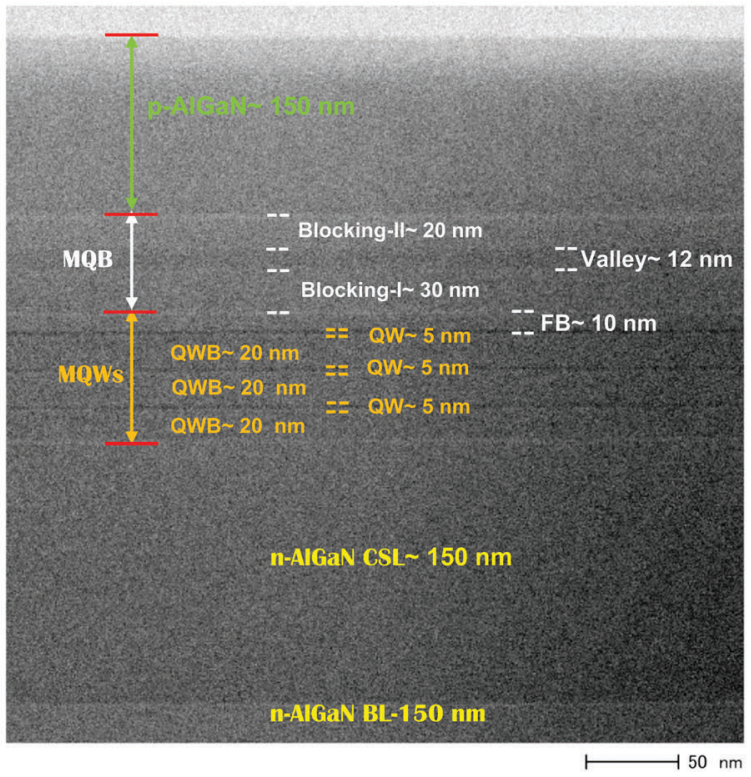

Fig. 4 (a) Cross-sectional HAADF-STEM images cut in the (10-10) zone axis tilted to $g=(0002)$ showing the screw type dislocations, (b) cross-sectional TEM images cut in the (10-10) zone axis tilted to the $g / 3 g, g=(10-20)$ zone axis showing the edge and mixed type dislocations (sample-A), and (c) magnified annular bright-field (ABF)-STEM images of point area "P1" taken from Fig. 3(a) are shown to show the approximate thickness of the device structure.

Table 1 Sample name, dislocation type, and dislocation density measured by high resolution scanning transmission electron microscopy (HR-STEM) bright and dark field observation

\begin{tabular}{lll}
\hline Sample name & Dislocation type & $\begin{array}{l}\text { Dislocation } \\
\text { density }\left(\mathrm{cm}^{-2}\right)\end{array}$ \\
\hline $310 \mathrm{~nm}$ UVB LED left & Total dislocation & $1.4 \times 10^{9}$ \\
side (sample-A) & Edge dislocation & $4.5 \times 10^{8}$ \\
& Mixed dislocation & $4.7 \times 10^{7}$ \\
$310 \mathrm{~nm}$ UVB LED right side & Screw dislocation & $5.5 \times 10^{7}$ \\
(reference) (sample-A) & Total dislocation & $1.1 \times 10^{9}$ \\
& Edge dislocation & $7.3 \times 10^{8}$ \\
& Mixed dislocation & $9.7 \times 10^{6}$ \\
& Screw dislocation & $5.7 \times 10^{7}$
\end{tabular}

Au layer (approximately 34\%). Therefore, we replaced the conventional Ni $(20 \mathrm{~nm}) / \mathrm{Au}(150 \mathrm{~nm})$ with a Ni $(1 \mathrm{~nm}) / \mathrm{Mg}(200 \mathrm{~nm})$ p-electrode, and the EQE was increased from $0.7 \%$ to $0.9 \%$, and, consequently, the output power also improved from $6.5 \mathrm{~mW}$ to $7.1 \mathrm{~mW}$, as shown in Fig. 5(a) and (b), respectively. This result confirmed the improvement in the LEE, which is caused by the highly reflective $\mathrm{Ni} / \mathrm{Mg}$ p-electrode and highly transparent p-AlGaN contact layer, as shown in Fig. 2. The current $v s$. voltage $(I-V)$ characteristics of sample-A with $\mathrm{Ni}(1 \mathrm{~nm}) / \mathrm{Mg}$ $(200 \mathrm{~nm})$ for the small current scale are given in the inset of Fig. 5(a), where high voltages were observed due to the device measurement under bare-wafer conditions. During the $I-V$ characterization, the turn on voltage of $8.0 \mathrm{~V}$ at $3 \mathrm{~mA}$ in sample-A 
(a)

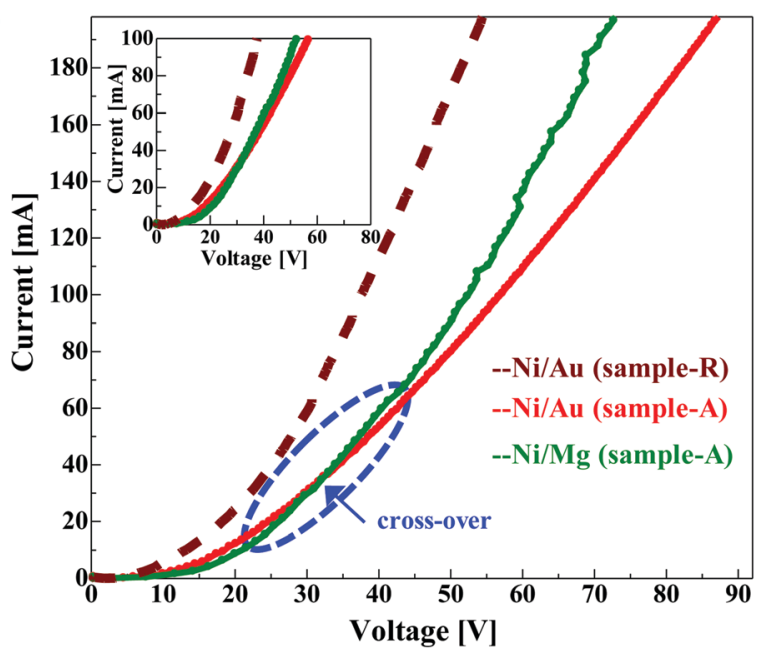

(b)

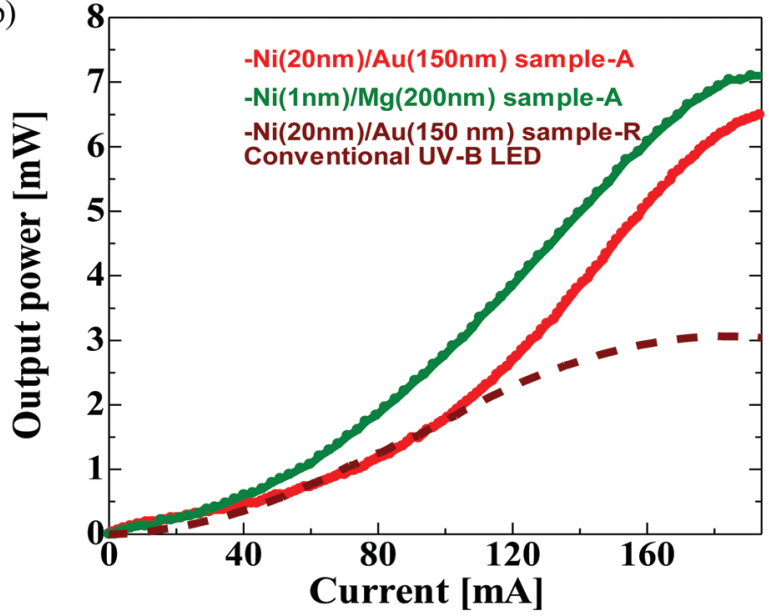

(c)

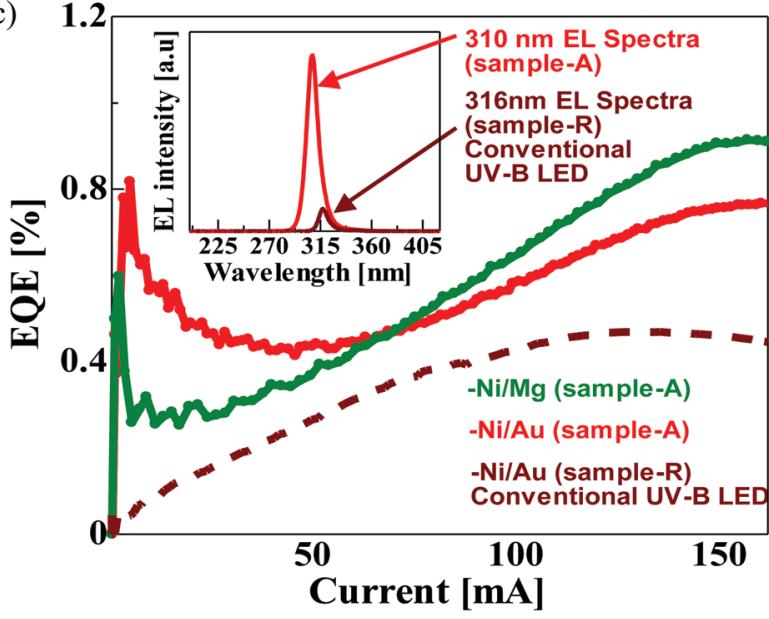

Fig. 5 UV-B LED sample-R with Ni/Au p-electrodes and sample-A both with $\mathrm{Ni} / \mathrm{Au}$ and with $\mathrm{Ni} / \mathrm{Mg}$ p-electrodes, measured at RT: (a) current vs. voltage $(I-V)$ characteristics (the $I-V$ characteristics under $100 \mathrm{~mA}$ is shown in the inset), (b) current vs. output power (I-L), and (c) current vs. EQE (I-EQE) characteristics under CW-operation under bare wafer conditions (wavelength vs. EL spectra are given in the inset).

with the $\mathrm{Ni} / \mathrm{Au}$ p-electrode and relatively high turn on voltage of 9.0 $\mathrm{V}$ at $3 \mathrm{~mA}$ in sample-A with $\mathrm{Ni} / \mathrm{Mg}$ were observed, as shown in the inset of Fig. 5(a). The Ni/Mg based p-electrode could encounter oxidation issues and the thin $\mathrm{MgO}$ layer could possibly give rise to high voltages in the UV-B LED devices. In the case of the UV-B LED (sample-A) with the Ni/Mg contact, the sum of n-contact resistance, $\mathrm{p}$-contact resistance and sheet resistance was found to be $<5 \Omega$. The specific contact resistivity of the $\mathrm{Ni} / \mathrm{Mg}$ electrode to the $\mathrm{p}-\mathrm{AlGaN}$ contact was difficult to measure separately at this time due to high $\mathrm{Al}$ contents as well as due to the degradation of the $\mathrm{Ni} / \mathrm{Mg}$ p-electrode, which is caused by annealing prior to measurement. Quite high voltages of $18.0 \mathrm{~V}, 24.0 \mathrm{~V}$ and $27.0 \mathrm{~V}$ at $20 \mathrm{~mA}$ were found for sample-R with $\mathrm{Ni} / \mathrm{Au}$, sample-A with $\mathrm{Ni} / \mathrm{Au}$ and sample-A with $\mathrm{Ni} / \mathrm{Mg}$ p-contact, respectively. The differential resistances at $198 \mathrm{~mA}$ are $5.5 \times 10^{-2} \Omega \mathrm{cm}^{2}, 7.3 \times 10^{-2} \Omega \mathrm{cm}^{2}$ and $8.6 \times 10^{-2} \Omega \mathrm{cm}^{2}$, respectively, for sample-R with $\mathrm{Ni} / \mathrm{Au}$, and sample-A with both $\mathrm{Ni} / \mathrm{Au}$ and $\mathrm{Ni} / \mathrm{Mg}$ p-contacts. One interesting cross-over behavior was observed in the $I-V$ characteristics of the sample-A with $\mathrm{Ni} / \mathrm{Mg}$ p-electrode, as shown in Fig. 5(a), and the reason for such abnormal behavior is still unknown. We speculate that it is caused by the reduction of electron injection efficiency (EIE) in the MQWs due to the thicker quantum barrier $(20 \mathrm{~nm})$ and low hole density in the p-AlGaN cladding layer. The UV-B LED is a bipolar device relying on the efficient injection of both minority carriers, and both holes and electrons need to be injected and distributed optimally in the active region (MQWs) to recombine for the effective operation of AlGaN UV-B LEDs. As shown in Fig. 4(c), a 20 nm-thick QWB can be seen clearly in the TEM image and the $20 \mathrm{~nm}$-thick QWB might cause the hole injection problem in the MQWs at low biased current, as shown in the (I-EQE) plot of Fig. 5(b). The possibility of Mg-diffusion as well as $\mathrm{H}$-atomic diffusion into the MQW region from p-AlGaN cladding layer side via un-doped AlGaN final barrier (FB) cannot be ignored. In particular, the $\mathrm{H}$-atoms can terminate to the $\mathrm{Mg}$-atomic bonds in $\mathrm{MQW}$ segion to make an $\mathrm{Mg}-\mathrm{H}$ complexes. But under the low biased current, the behavior of $\mathrm{Mg}-\mathrm{H}$ complexes in the MQW region could be an interesting phenomenon. We still do not know the most concrete reason for this abnormal behavior of the $I$ vs. EQE (I-EQE) characteristic in sample-B.

One important result in the application of UV-B LEDs came from Boston University in the USA, where it was discovered that a UV-B LED with an emission wavelength of $293 \mathrm{~nm}$ is 2.4 times more efficient than sunlight for producing vitamin D3 in human skin. ${ }^{18}$ But, the light extraction efficiency (LEE) was found to be very low in the p-GaN based UV-B LED devices due to the light absorption by p-GaN. ${ }^{6,27}$ In this work, we successfully achieve more than $97 \%$ transmittance from the p-AlGaN contact layer with the UV-B LED, as shown in Fig. 2. The IQE of the $295 \mathrm{~nm}$-UV-B LED (sample-B) was measured by excitationpower-density-dependent and temperature-dependent PL spectroscopy, as shown in Fig. 6(a) and (b), using the procedure given in ref. 33. Fig. 6(a) shows the IQE curves at $10 \mathrm{~K}$ (black circles) and RT (red circles) for UV-B LED sample-B as a function of excitation power density under the excitation wavelength of $240 \mathrm{~nm}$. For this sample, the IQE curves were normalized by the maximum IQE at $10 \mathrm{~K}$. From this normalization, the maximum IQE at RT is estimated to be $47 \%$. Fig. 6(b) shows the integrated 
(a)

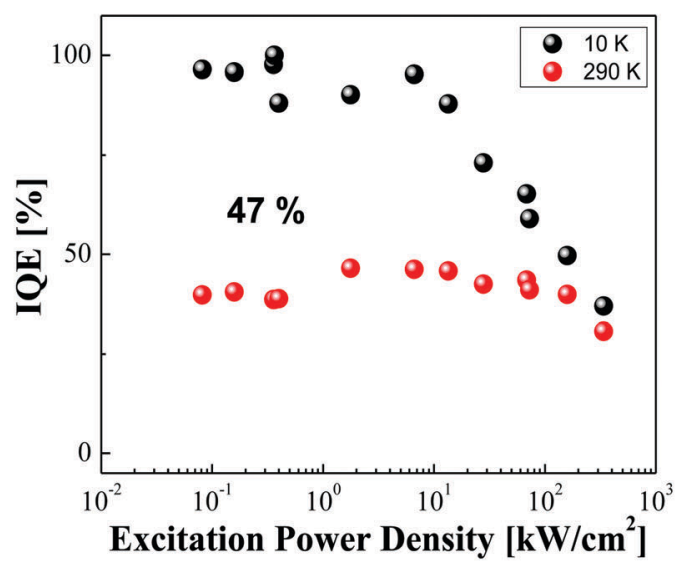

(c)

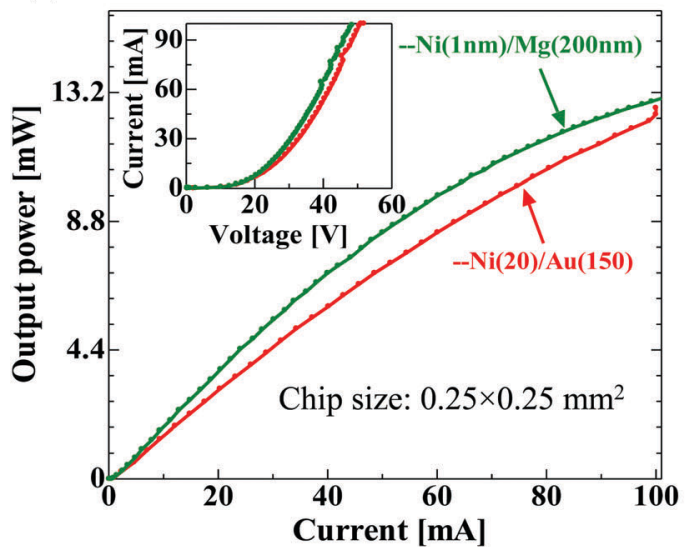

(b)

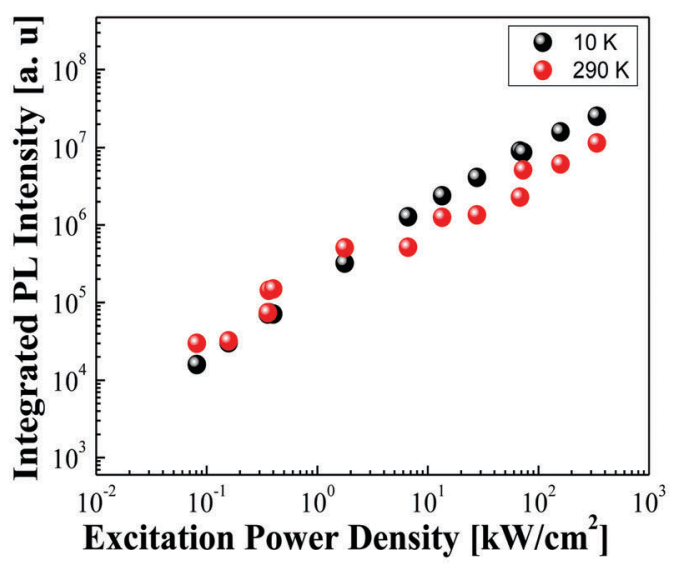

(d)

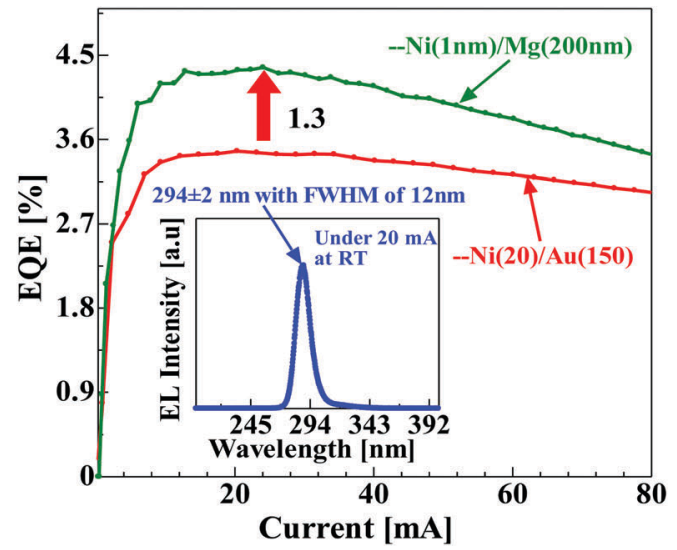

Fig. 6 (a) Excitation power density vs. IQEs measured at $10 \mathrm{~K}$ and $290 \mathrm{~K}$ for sample-B, and (b) temperature-dependence of PL as a function of excitation power density, (c) current vs. output power (I-L) (current vs. voltage (I-V) characteristics are given in the inset), and (d) current vs. EQE (I-EQE) characteristics of $294 \mathrm{~nm}$ UV-B LEDs with both Ni/Au and Ni/Mg p-electrodes under CW-operation under bare wafer conditions (sample-B) (wavelength vs. EL spectrum is given in the inset).

PL intensity curves at $10 \mathrm{~K}$ (black circles) and RT (red circles) for UV-B LED sample-B as a function of excitation power density under the excitation wavelength of $240 \mathrm{~nm}$. For this sample, the IQE curves were normalized by the maximum IQE at $10 \mathrm{~K}$. We also observed that the IQE at a lower excitation power density remains constant, as shown in Fig. 6(a). This observation indicates that nonradiative recombination centers freeze at $10 \mathrm{~K}$, which is in close agreement with ref. 5, 6 and 33. After the IQE enhancement, the UV-B LED (sample-B) device was tested with an emission wavelength of $295 \mathrm{~nm}$. The output power and EQE for sample-B are shown in Fig. 6(c) and (d), respectively. The $I-V$ characteristics and EL spectrum, respectively, of sample-B device are shown in the insets of Fig. 6(c) and (d). High voltages were observed due to the low conductivity of the p-AlGaN contact layer, as well as measurement under bare-wafer conditions. During the $I-V$ characterization, a turn on voltage of $6.0 \mathrm{~V}$ at $1 \mathrm{~mA}$ in sample-B with the $\mathrm{Ni} / \mathrm{Au}$ p-electrode and nearly the same turn on voltage of $5.0 \mathrm{~V}$ at $1 \mathrm{~mA}$ in sample-B with $\mathrm{Ni} / \mathrm{Mg}$ were observed, as shown in the inset of Fig. 6(c). Now, the operational voltages at $100 \mathrm{~mA}$ are $45.0 \mathrm{~V}$ and $50.0 \mathrm{~V}$, respectively, for sample-B with the Ni/Au and Ni/Mg p-electrodes, as shown in the inset of Fig. 6(c). In the case of sample-B, both the turn on voltages and the operational voltages were found to be lower than those of sample-A.

After the MQW optimization for $295 \mathrm{~nm}$ emission, as shown in Fig. 6(d), a record EQE of $4.4 \%$ at $20 \mathrm{~mA}$ dc drive under $\mathrm{CW}$-operation with a maximum output power of $13 \mathrm{~mW}$ was successfully realized under bare-wafer conditions, as shown in Fig. 6(c) and (d) respectively. The LEE enhancement factor of 1.3 was realized, as shown in Fig. 6(d), which was caused by the reflectivity enhancement from the $\mathrm{Ni} / \mathrm{Mg}$ p-electrodes. The LEE enhancement is attributed to the improvement in the reflectivity from approximately $34 \%(\mathrm{Ni} / \mathrm{Au})$ to $88 \%(\mathrm{Ni} / \mathrm{Mg})$ and also to the highly transparent p-ALGaN contact layer, as shown in Fig. 2 and 6(d). Further improvements in the device performance can be made by increasing the EIE through optimization of the MQB or using polarization doping on the p-AlGaN side to reduce the specific contact resistances. In particular, the IQE of the UV-B LED devices can further be improved from 50\% (this work) to $60 \%$ by reducing the TDDs both in the n-AlGaN CSL 
and in MQWs grown on the AlN template on the overlayer of a nano-PSS. One very big issue for UV-B LEDs is their low performance as compared to visible or blue LEDs. ${ }^{5,6,19,20,34}$ Previously, the LEE was significantly improved by introducing a highly transparent (92\%) p-AlGaN contact layer and a highly reflective Rh electrode along with lens-like resin encapsulation on a microPSS. $^{20}$ This structure could also help us to combat the increase in forward voltage with $\mathrm{p}$-AlGaN, ${ }^{5,6,20,27,28,35}$ if we were to use it in the UV-B LED. One other important idea to further improve the performance of narrow band UV-B LEDs, especially the LEE, is to use a reflective photonic crystal (PhC) for the p-type AlGaN layer as it was used for the UVC LED. ${ }^{19}$ By combining transparent $\mathrm{p}$-AlGaN contact layers with a $\mathrm{PhC}$ as well as a highly reflective $\mathrm{Ni} / \mathrm{Al}$ p-electrode or $\mathrm{Rh}$ p-electrode, along with further reductions in the TDDs (100 arcsec) of AlN templates grown on a nano-PSS, we can possibly make very large increases in the IQEs up to $60-70 \%$ for UV-B emission. The improved performance of $13 \mathrm{~mW}$ power, with $295 \mathrm{~nm}$-emission wavelength UV-B light sources, which can be used to produce vitamin D3 in the human body, and $310 \mathrm{~nm}$-emission, which is the ideal wavelength to induce HBGT-1 in the human body to treat psoriasis, as well as being equally useful for enriching phytochemicals in plants, would have an impact on QOL both through applications in medical science and in agricultural science.

\section{Conclusions}

We successfully fabricated AlGaN based UV-B LEDs on AlN/sapphire templates in the LP-MOVPE system. We successfully combined a graded AlGaN buffer layer, AlGaN MQWs with high IQE, a transparent p-AlGaN contact layer, and a highly-reflective p-type electrode for the operation of UV-B LED. The screw type TDDs were reduced to $5.5 \times 10^{7} \mathrm{~cm}^{-2}$ in the current spreading layer. Using optimized growth conditions of epi-layers, we demonstrated an output power of $7.1 \mathrm{~mW}$ for an UV-B LED operating at $310 \mathrm{~nm}$ using a bare-wafer level measurement. The LEE was increased by introducing a highlyreflective Ni/Mg p-type electrode. We also demonstrated a high IQE of $47 \%$ for AlGaN UV-B LED emission at $295 \mathrm{~nm}$, by reducing the TDDs using a graded n-AlGaN buffer layer. Finally, we achieved a record EQE of $4.4 \%$ under a dc drive current of $20 \mathrm{~mA}$ and the maximum output power of $13 \mathrm{~mW}$ for a $295 \mathrm{~nm}$-emission UV-B LED using a highly reflective Ni/Mg p-electrode. Both $310 \mathrm{~nm}$-and 295 nm-emission UV-B LED devices are very useful for both medical and agricultural applications.

\section{Conflicts of interest}

There are no conflicts to declare.

\section{Acknowledgements}

This work was supported in part by the New Energy and Industrial Technology Development Organization (NEDO). We would like to thank the SPDR office of RIKEN for their continued financial support to make this research possible.

\section{References}

1 M. D. Daniel, et al., A History of photodynamic therapy, Aust. N. Z. J. Surg., 1991, 61, 340-348.

2 J. A. Parrish, et al., Action spectrum for phototherapy of psoriasis, J. Invest. Dermatol., 1981, 76, 359-362.

3 P. J. Hargis Jr, et al., Ultraviolet fluorescence detection and identification of protein, DNA, and bacteria, Proc. SPIE, 1995, 147, 2366.

4 Retrieved on Oct 05, Semicond. Today, www.semiconductortoday.com, 2015, 10(1), 80.

5 M. Kneissl and J. Rass, et al., III-Nitride Ultraviolet Emitters. Springer Series in Material Science 227, Woodhead Publishing, 2016, ISSN 0933-033X.

$6 \mathrm{H}$. Hirayama, et al., Recent progress and future prospects of AlGaN based high-efficiency deep-ultraviolet light-emitting diodes, Jpn. J. Appl. Phys., 2014, 53, 100209.

7 U. Wollina, et al., Psoroatic arthritis, Dermatol. Ther., 2010, 23, 123-136.

8 A. Nast, et al., S3 - Guidelines on the treatment of psoriasis vulgaris (English version), J. Dtsch Dermatol. Ges., 2012, 10, S1-95.

9 E. Verhaeghe, et al., Intrapatient comparison of 308-nm monochromatic excimer light and localized narrow-band UVB phototherapy in the treatment of vitiligo: a randomized controlled trial, Dermatology, 2011, 223, 343-348.

10 W. L. Morison, et al., Phototherapy and Photochemotherapy of Skin Disease, Raven Press, New York, 2nd edn, 1991.

11 P. E. Hockberger, et al., A history of ultraviolet photobiology for humans, animals and microorganisms, Photochem. Photobiol., 2002, 76(6), 561-579.

12 D. J. William, et al., Clinical Dermatology, Saunders Elsevier, p. 335, ISBN 0-7216-2921-0(2006).

$13 \mathrm{~K}$. Torii, et al., UVB irradiation induces HMGB1 expression in keratinocytes without promoting apoptosis, Exp. Dermatol., 2016, 25(9), 741-742.

14 L. Kemény, et al., Ultraviolet B treatment of plaque-type psoriasis using light-emitting diodes: a new phototherapeutic approach, J. Am. Acad. Dermatol., 2009, 60, AB8.

15 L. Kemény, et al., Targeted phototherapy of plaque type psoriasis using UVB-LEDs, Br. J. Dermatol., 2010, 163, 167-173.

16 J. C. Beani, et al., Narrow-band UVB therapy in psoriasis vulgaris: good practice guideline and recommendations of the French Society of Photodermatology, Ann. Dermatol. Venereol., 2010, 137, 21-31.

17 S. H. Ibbotson, et al., An update and guidance on narrowband ultraviolet B phototherapy, Br. J. Dermatol., 2004, 151(2), 283-297.

18 T. A. Kalajian, et al., Ultraviolet B Light Emitting Diodes (LEDs) Are More Efficient and Effective in Producing Vitamin D3 in Human Skin Compared to Natural Sunlight, Sci. Rep., 2017, 7, 11489.

19 Y. Kashima, et al., High external quantum efficiency (10\%) AlGaN based deep-ultraviolet light-emitting diodes achieved by using highly reflective photonic crystal on p-AlGaN contact layer, Appl. Phys. Express, 2018, 11, 012101. 
20 T. Takano, et al., Deep-ultraviolet light-emitting diodes with external quantum efficiency higher than $20 \%$ at $275 \mathrm{~nm}$ achieved by improving light-extraction efficiency, Appl. Phys. Express, 2017, 10(3), 031002.

21 C. Pernot, et al., Improved Efficiency of 255-280 nm AlGaNBased Light-Emitting Diodes, Appl. Phys. Express, 2010, 3, 061004.

22 S. Hwang, et al., $276 \mathrm{~nm}$ Substrate-Free Flip-Chip AlGaN Light-Emitting Diodes, Appl. Phys. Express, 2011, 4, 032102.

23 A. Fujioka, et al., Improvement in Output Power of 280-nm Deep Ultraviolet Light-Emitting Diode by Using AlGaN Multi Quantum Wells, Appl. Phys. Express, 2010, 3, 041001.

24 M. Ippommatsu, et al., Optronics, 2014, 2, 71.

25 K. H. Kim, et al., AlGaN-based ultraviolet light-emitting diodes grown on AlN epilayers, Appl. Phys. Lett., 2004, 85, 4777.

26 N. Susilo, et al., Effect of the GaN:Mg Contact Layer on the Light-Output and Current-Voltage Characteristic of UVB LEDs, Phys. Status Solidi A, 2017, 1700643.

27 M. Jo, et al., Enhanced light extraction in $260 \mathrm{~nm}$ lightemitting diode with a highly transparent p-AlGaN layer, Appl. Phys. Express, 2016, 9, 012102.

28 N. Maeda, et al., Enhancing the light-extraction efficiency of AlGaN deep-ultraviolet light-emitting diodes using highly reflective $\mathrm{Ni} / \mathrm{Mg}$ and $\mathrm{Rh}$ as p-type electrodes, Jpn. J. Appl. Phys., 2018, 57, 04FH08.

29 N. Maeda, et al., Efficiency improvement of AlGaN UVC-LEDs using highly-reflective Ni/Al p-type electrode, Ext. Abstract of ISCS 2017, D5.6, Berlin, Germany, May 14-18, 2017.

30 James R. Grandusky, et al., 270 nm Pseudomorphic Ultraviolet Light-Emitting Diodes with Over $60 \mathrm{~mW}$ Continuous Wave Output Power, Appl. Phys. Express, 2013, 6, 032101.

31 H. Hirayama, et al., 231-261 nm AlGaN deep-ultraviolet light-emitting diodes fabricated on AlN multilayer buffers grown by ammonia pulse-flow method on sapphire, Appl. Phys. Lett., 2007, 91, 071901.

32 T. Nishida, et al., Submilliwatt operation of AlGaN-based ultraviolet light-emitting diode using short-period alloy superlattice, Appl. Phys. Lett., 2001, 78, 399.

33 Y. Yamada, et al., Internal Quantum Efficiency and Nonradiative Recombination Rate in InGaN-Based NearUltraviolet Light-Emitting Diodes, Jpn. J. Appl. Phys., 2012, 51, 072102.

34 M. Katsuragawa, et al., Thermal ionization energy of Si and Mg in AlGaN, J. Cryst. Growth, 1998, 528, 189-190.

35 M. Marques, et al., Influence of miscibility on the energy-gap dispersion in $\mathrm{Al}_{\mathrm{x}} \mathrm{Ga}_{1-\mathrm{x}} \mathrm{N}$ alloys: First-principles Calculations, Phys. Rev. B: Condens. Matter Mater. Phys., 2007, 75, 033201. 